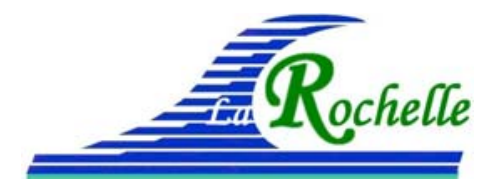

XV $V^{\text {èmes }}$ Journées Nationales Génie Côtier - Génie Civil

La Rochelle, 29 au 31 mai 2018

DOI:10.5150/jngcgc.2018.092 (C) Editions Paralia CFL

disponible en ligne - http://www.paralia.fr - available online

\title{
Intérêt du "Data-Rescue" en marégraphie : des archives papiers à l'étude de l'évolution du niveau marin. Exemple de la reconstruction de la série marégraphique de Saint-Nazaire depuis le 19ème siècle.
}

\author{
Yann FERRET ${ }^{1,3}$, Swann LORRETTE-RIBEROLLES ${ }^{2,3}$, Vincent DONATO ${ }^{1,3}$, \\ Nicolas POUVREAU ${ }^{1,3}$
}

1. Shom. 13 rue du Châtellier, CS 92803, 29228 BREST CEDEX 2, France.

2. UMR 7266 LIENSs, CNRS/Université de la Rochelle, 2 rue Olympe de Gouges, La Rochelle 17000, France

3. IR-ILICO, réseau SONEL (Infrastructure de recherche littorale et côtière, Système d'observation du niveau des eaux littorales)

\section{Résumé :}

La hausse du niveau de la mer liée aux changements climatiques globaux demeure l'une des préoccupations sociétales majeures. L'analyse des données marégraphiques, constituant souvent les seules informations disponibles à l'échelle des décennies, voire des siècles passés, se révèle être un moyen idéal pour parvenir à dégager des tendances sur le long terme. Or, malgré un patrimoine historique marégraphique français conséquent, la majorité de ces mesures est encore sous forme papier et n'est donc pas exploitée. Dans ce contexte, un important travail de "Data rescue", ou sauvetage des données historiques, est entrepris au Shom depuis quelques années afin d'inventorier et de valoriser ses archives marégraphiques historiques.

L'exemple de la reconstruction de la série marégraphique de Saint-Nazaire est présenté pour illustrer les étapes nécessaires à ce type de travail. Les données du marégraphe ont été numérisées, contrôlées, corrigées et validées, ce qui a abouti à la constitution d'une série de hauteurs d'eau horaires couvrant une période de plus de 150 ans (1863aujourd'hui). Cette série est globalement de bonne qualité (seules $16 \%$ des données digitalisées qualifiées de "potentiellement de mauvaise qualité") et est caractérisée par une tendance à l'augmentation des niveaux moyens à Saint-Nazaire depuis 1863 de l'ordre de $+1.03 \pm 0.05 \mathrm{~mm} / \mathrm{an}$.

\section{Mots-clés :}

Data-Rescue, Marégraphie, Données historiques, Niveau marin, Saint-Nazaire.

\section{Introduction}

L'étude du changement climatique global et son influence sur les variations du niveau marin selon différentes échelles temporelles, qu'il s'agisse de l'évolution du niveau moyen de la mer ou de l'évolution des évènements extrêmes, constitue un enjeu actuel fort pour les sociétés humaines. Depuis les années 1990, les données altimétriques 


\section{Thème 7 - Risques côtiers}

satellitaires permettent d'avoir une vision globale de ces changements et rendent compte de l'hétérogénéité spatiale des variations du niveau marin (e.g. CAZENAVE \& LLOVEL, 2010). Les technologies embarquées pour réaliser ces études, et les traitements appliqués aux données ne cessent de s'améliorer, mais il est toujours nécessaire de croiser ces mesures avec les observations issues des marégraphes ponctuels, particulièrement dans les zones côtières (CIPOLLINI et al., 2017). Les mesures effectuées à l'aide de marégraphes sont pratiquement les seules archives disponibles nous renseignant sur l'évolution du niveau marin historique, à l'échelle des décennies, voire des siècles passés. Il est admis qu'au moins 60 ans d'enregistrement sont raisonnables pour estimer les variations eustatiques actuelles et filtrer les contributions cycliques et irrégulières du signal marégraphique (DOUGLAS, 1991), d'où la nécessité d'avoir des séries marégraphiques les plus longues possibles.

Dès le milieu du 19ème siècle, les hauteurs d'eau sont mesurées de manière systématique et continue en de nombreux sites le long des côtes françaises grâce à l'utilisation de marégraphes. Or, un état des lieux des données historiques du niveau marin met en évidence le fait que la majorité de ces mesures sont encore sous forme papier et ne sont donc pas exploitées (POUVREAU, 2008). En France, les seules séries d'une durée supérieure à cent ans disponibles au format numérique sont celles de Brest (POUVREAU, 2008), de Marseille (WÖPPELMANN et al., 2014) et la série composite du Pertuis d'Antioche (GOURIOU et al., 2013).

Dans ce contexte, un important travail de "Data rescue", ou sauvetage des données historiques, est entrepris au Shom afin de sauvegarder les nombreuses données historiques encore au format papier. Ces projets ont pour objectif à terme de pérenniser l'ensemble des documents historiques conservé dans ses archives afin de permettre l'amélioration de la connaissance des évolutions historiques des zones côtières (LATAPY et al., 2017), du niveau moyen de la mer, etc. Cette initiative s'intègre dans une démarche actuelle plus internationale et répond aux recommandations du programme Global Sea Level Observing System (GLOSS) piloté par la Commission Océanographique Intergouvernementale (COI) de l'UNESCO sur la valorisation des observations "archéologiques" de niveaux d'eau (BRADSHAW et al., 2015).

L'objectif de cet article est de présenter les réalisations actuelles au Shom en terme de Data Rescue en marégraphie afin de rendre compte du fort potentiel scientifique des documents d'archives papiers. L'exemple du travail de reconstruction réalisé pour la série marégraphique historique de Saint-Nazaire (FERRET, 2016) sera présenté pour illustrer ce propos.

\section{2. "Data Rescue" en marégraphie : redécouverte de données inédites pour l'étude du niveau marin}

La première étape primordiale de ce travail de Data rescue est la réalisation d'un inventaire le plus exhaustif possible (lieu de mesure, période, type de 


\section{XVèmes Journées Nationales Génie Côtier - Génie Civil \\ La Rochelle, 29 au 31 mai 2018}

documents/données, ...) de l'ensemble des données analogiques disponibles afin de permettre l'estimation de leur potentiel scientifique. La difficulté de ce travail réside dans le fait que ces documents historiques ont été disséminés au cours du temps et ne sont donc pas concentrés en un lieu unique (POUVREAU, 2008).

De par son histoire, le Shom dispose d'un important patrimoine technique et historique, notamment dans le domaine de la marégraphie (dès le début du $19^{\text {ème }}$ siècle). L'ensemble des mesures conservées aux archives du Shom concerne principalement des ports français, mais également de manière plus ponctuelle dans le temps des sites localisés dans les différentes mers du globe. Depuis quelques années, un effort important est fait pour valoriser ces documents longtemps restés oubliés, notamment grâce à des financements provenant du MTES/DGPR (Ministère de la transition écologique et solidaire / Direction générale de la prévention des risques) et dans le cadre de l'IR ILICO - SONEL. Une réflexion est menée pour permettre la mise à disposition prochaine de l'inventaire de ces archives, actuellement en cours de finalisation.

La figure 1 illustre ce que pourrait être l'état des séries marégraphiques disponibles si l'ensemble des données papiers des archives du Shom (en cours d'inventaire) étaient numériques. L'évaluation des tendances séculaires du niveau marin pourrait ainsi être réalisée en de nombreux sites français. Ce constat est d'autant plus vrai si ce travail d'inventaire est généralisé à d'autres centres d'archives qui peuvent également contenir des documents en relation avec les mesures marégraphiques et ainsi venir compléter ces séries historiques (ex : série de Saint-Nazaire, Figure 1).

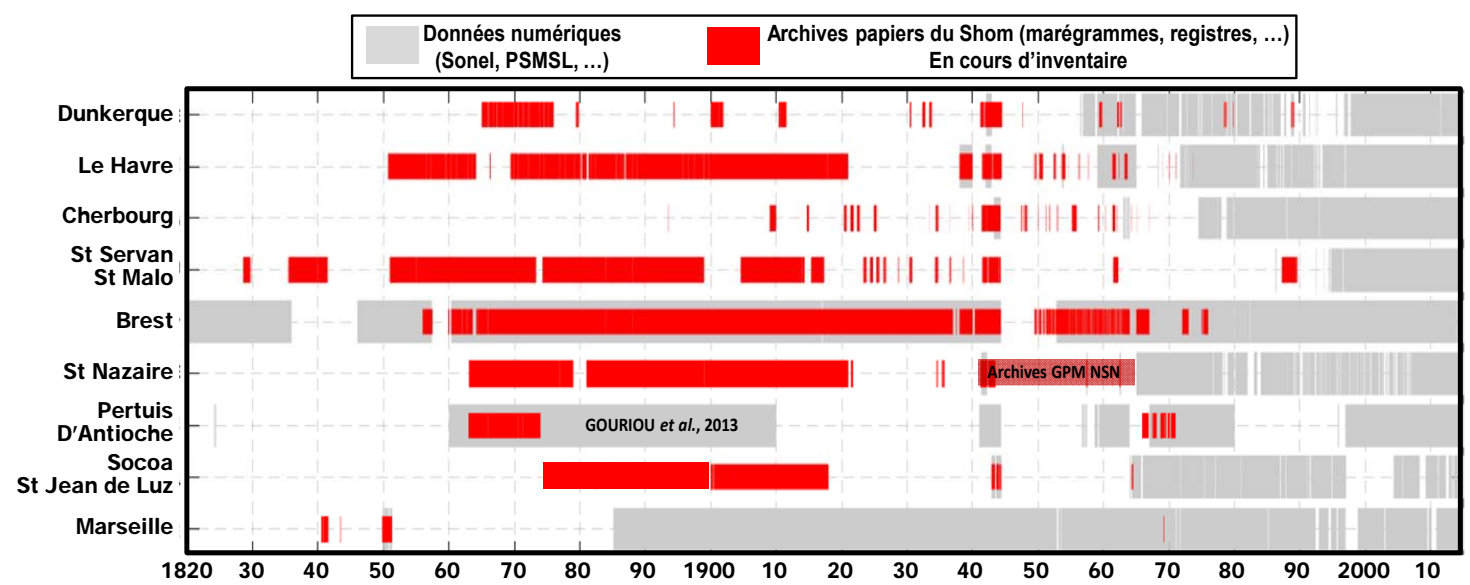

Figure 1. Exemples de disponibilité numérique de séries marégraphiques historiques françaises (sources SONEL, PSMSL, ...) et illustration du potentiel des archives papier du Shom (non exhaustif, inventaire en cours).

Les documents historiques recherchés correspondent à des mesures marégraphiques (registres de marée, marégrammes), mais également à des documents d'archives apportant des informations relatives à ces dites mesures (métadonnées contextuelles). 


\section{Thème 7 - Risques côtiers}

Ces métadonnées constituent une source d'information essentielle pour connaitre l'historique de l'observatoire de marées d'un lieu (matériel utilisé, suivi du zéro instrumental, ...), et ainsi augmenter la cohérence et la qualité de la série finale.

Une fois les documents identifiés, ils doivent être numérisés. L'exemple du travail effectué sur les observations marégraphiques historiques de Saint-Nazaire présenté ciaprès détaille brièvement l'ensemble des différentes étapes nécessaire pour rendre ces données exploitables et pour étudier l'évolution des composantes du niveau de la mer.

\section{Exemple de la reconstruction de la série marégraphique de Saint-Nazaire (1821 - aujourd'hui)}

3.1 Méthodologie

Le travail se décompose en 3 actions principales : la recherche des données et d'archives documentaires, la numérisation des données, et la mise en cohérence des données et leur validation.

La totalité des principaux centres d'archives identifiés comme disposant des documents en lien avec la mesure marégraphique à Saint-Nazaire a été consultée : les archives du Shom, les archives du Grand Port Maritime -Nantes-Saint-Nazaire (GPM-NSN), les archives des Services Historiques de la Défense (SHD) de Rochefort et de Vincennes, les archives départementales de Loire-Atlantique. Les plus anciennes observations marégraphiques retrouvées pour Saint-Nazaire datent de 1821 (lecture à une échelle de marée pendant quelques mois) et les premières mesures systématiques du niveau marin y sont effectuées à partir de 1863 grâce à l'installation d'un marégraphe à flotteur. Ainsi, la totalité des documents marégraphiques permet de couvrir une période d'environ 195 ans et de reconstruire au moins 125 ans de mesures continues du niveau d'eau à Saint-Nazaire. La collaboration du GPM-NSN a été essentielle et très bénéfique à l'étude: l'intégralité des marégrammes et documents relatifs à Saint-Nazaire disponibles dans leurs archives (1932-2000) a été mise à notre disposition (Figure 1).

La deuxième étape du travail a consisté à digitaliser ces données. L'ensemble des registres de marée (tableaux de valeurs) a été numérisé: ce sont plus de 500.000 valeurs de hauteurs d'eau qui ont été numérisées manuellement selon un pas de temps horaire, soit 59 ans de mesures $(1863$ - 1920). Les marégrammes ont nécessité d'être scannés avant l'extraction des mesures de hauteurs d'eau en fonction du temps. Par la suite, ils ont été digitalisés grâce au logiciel NUNIEAU développé et mis librement à disposition par le CEREMA (PONS, 2008) dans le but d'extraire les données de niveaux d'eau en fonction du temps. L'ensemble des marégrammes inédits (1932-1965) a été digitalisé, ce qui correspond au traitement d'environ 500 documents et à l'extraction de plus de 8000 courbes journalières.

A l'issue de la digitalisation de ces données, ces dernières doivent être validées. Cette étape a pour objectifs de rendre la série reconstruite cohérente en temps (exprimée en TU) et en hauteur (exprimée selon le zéro hydrographique redéfini en 1996) et de 


\section{XVèmes Journées Nationales Génie Côtier - Génie Civil \\ La Rochelle, 29 au 31 mai 2018}

qualifier les mesures inédites en fonction de leur qualité (Figure 2). Pour cela, il a été nécessaire de prendre en considération l'ensemble des métadonnées relatives aux mesures marégraphiques disponibles et des procédures ont été adaptées ou développées pour permettre de détecter et corriger/supprimer les anomalies dans les mesures.

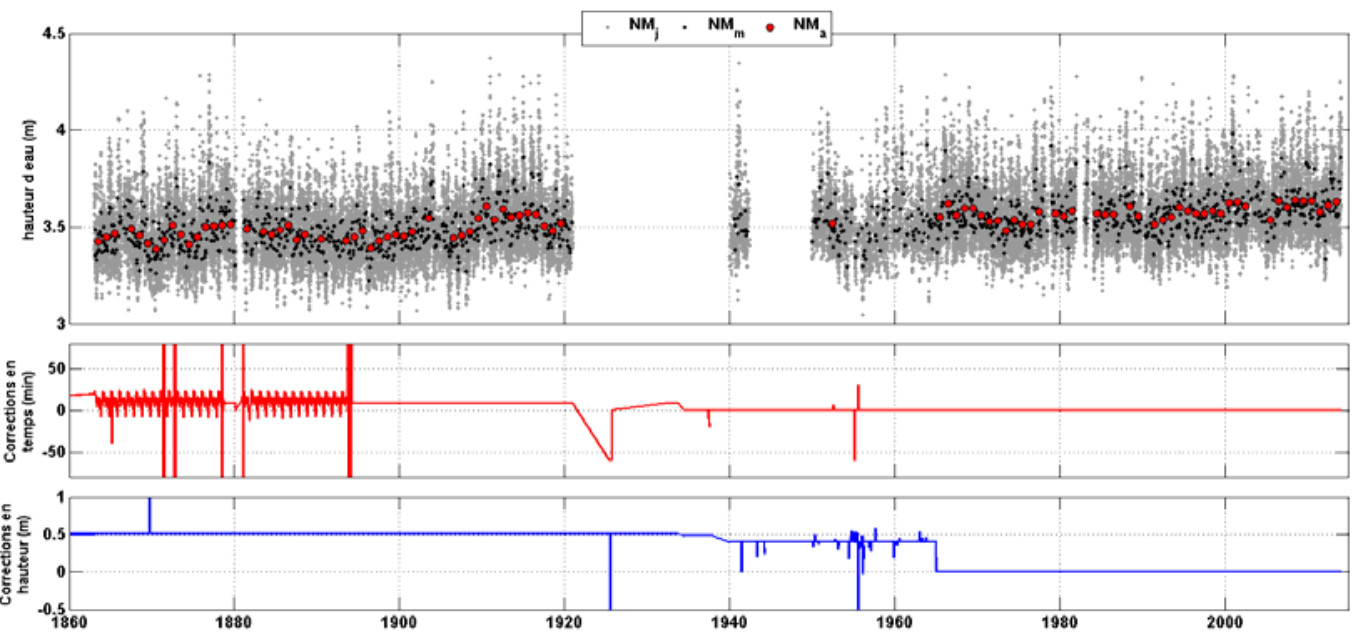

Figure 2. Calcul des niveaux moyens journaliers (NMj), mensuels (NMm) et annuels (NMa) à Saint-Nazaire à partir de la série marégraphique reconstruite qualifiée (haut)

; corrections temporelles (milieu) et en hauteurs (bas) appliquées (Les périodes en bleu : données déjà numériques qui n’ont pas fait l'objet de contrôle durant ce travail).

La qualification de ces données est une étape nécessaire avant toute analyse ultérieure (évolution du niveau de la mer, niveaux extrêmes...). Cette action a été réalisée grâce à l'analyse des résidus en temps et en hauteurs des PM et BM : les résidus correspondent aux différences entre les niveaux d'eau observés pour une $\mathrm{PM} / \mathrm{BM}$ et ceux prédits pour cette même PM/BM. Cette méthode permet d'identifier des sauts verticaux ou les décalages temporels présents dans les données reconstruites. Des comparaisons interséries ont également été faites. En effet, des sites proches géographiquement sont régulièrement soumis aux mêmes conditions atmosphériques à large échelle, ce qui induit normalement une bonne corrélation des variations journalières et mensuelles du niveau marin observées sur ces différents sites. Ainsi, cela permet de mettre en évidence les périodes pour lesquelles la qualité de la donnée digitalisée est potentiellement suspecte. La qualification finale correspond à une inspection visuelle des données.

Cela a permis d'identifier les périodes pour lesquelles les données étaient de mauvaise qualité (environ $16 \%$ des données numérisées). Il a ainsi été possible de mettre en évidence que l'observatoire des marées de Saint-Nazaire était régulièrement soumis à un envasement de la base du puits de tranquillisation et d'identifier des périodes pour lesquelles les données n'étaient certainement pas bien référencées verticalement (19351937). A partir de ces données validées, les niveaux moyens journaliers, mensuels et 


\section{Thème 7 - Risques côtiers}

annuels ont pu être calculés (Figure 2), selon les recommandations du PSMSL (Permanent Service for Mean Sea Level, http://www.psmsl.org/data/supplying/).

\subsection{Résultats}

La reconstruction de la série historique de hauteurs d'eau mesurées à Saint-Nazaire depuis le milieu du 19 ème siècle permet d'appréhender l'évolution séculaire du niveau marin pour ce secteur, ce qui était jusqu'alors impossible (Figure 3).

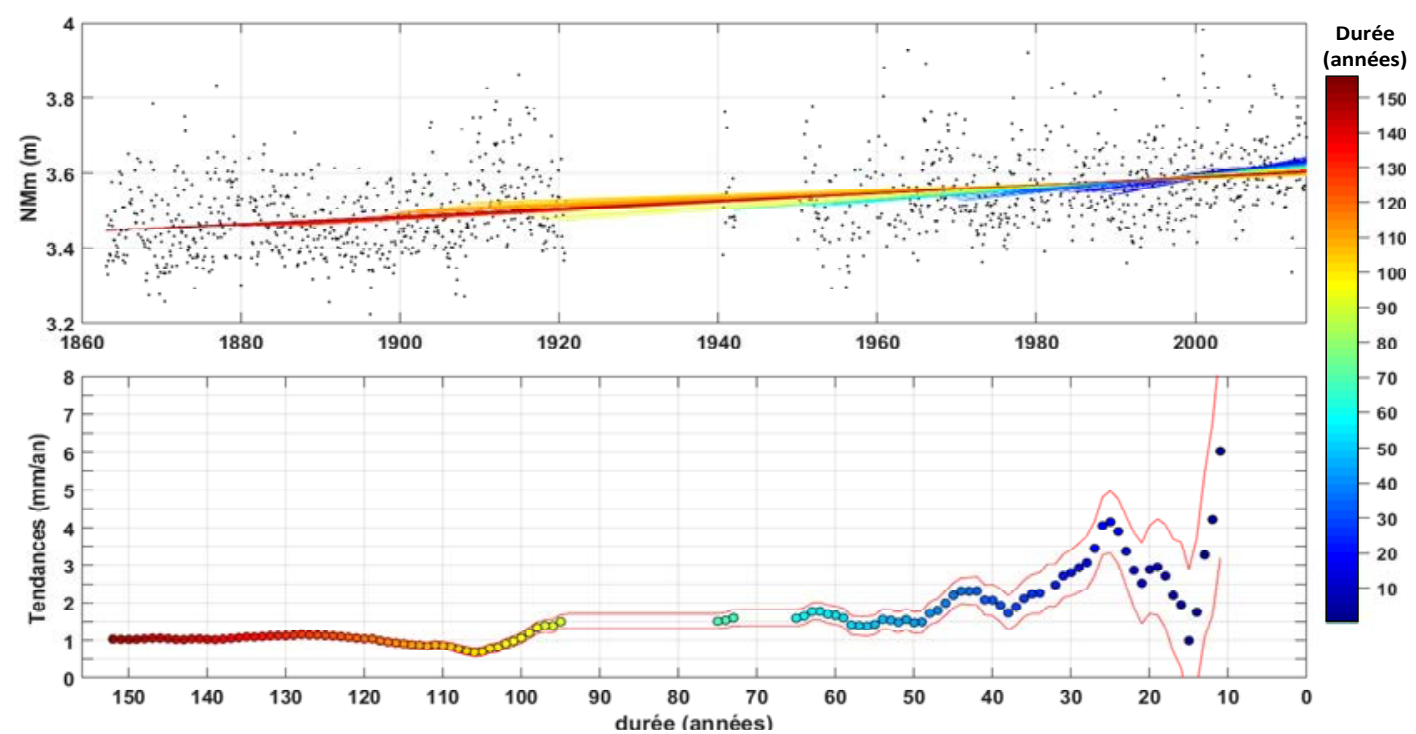

Figure 3. Evolution des niveaux moyens mensuels (NMm) calculés à partir de la série marégraphique historique de Saint-Nazaire (haut). Calcul des tendances linéaires à partir de 2014 et de l'erreur type associée en fonction de la durée d'observation (bas).

En fonction de la durée considérée pour estimer l'évolution du niveau marin à SaintNazaire, il est possible d'observer des variations dans les valeurs calculées (Figure 3). Il est à noter que les valeurs obtenues sur la période [1993 - 2014] sont cohérentes avec les valeurs déduites des mesures satellites récentes (CAZENAVE \& LLOVEL, 2010), étant de $2.87 \pm 1 \mathrm{~mm} /$ an et $3.4 \pm 0.4 \mathrm{~mm} /$ an respectivement. Les erreurs type relativement importantes calculées sur ces périodes courtes s'expliquent par le plus faible nombre de niveaux moyens mensuels considéré dans le calcul de ces tendances.

Les tendances calculées à partir des années $1950(+1.64 \pm 0.2 \mathrm{~mm} / \mathrm{an})$ sont cohérentes avec celles calculées à Brest et sur le littoral charentais.

Entre 1863 et 2014, une augmentation globale du niveau moyen de la mer de l'ordre de $+1.03 \pm 0.05 \mathrm{~mm} /$ an est observée à Saint-Nazaire. Ce résultat tend à être plus faible en comparaison de ce qui est rapportée dans la littérature pour des périodes similaires : entre 1860 et 2010 , la tendance estimée à Brest est de $+1.32 \pm 0.07 \mathrm{~mm} / \mathrm{an}$, celle de la série composite du Pertuis d'Antioche est de $+1,38 \pm 0.07 \mathrm{~mm} /$ an et celle de Liverpool estimée à $+1.44 \pm 0.13 \mathrm{~mm} / \mathrm{an}$ (GOURIOU, 2012). Cette faible évolution de la 


\section{XVèmes Journées Nationales Génie Côtier - Génie Civil \\ La Rochelle, 29 au 31 mai 2018}

tendance séculaire à Saint-Nazaire pourrait être réelle, mais pourrait également être due à des problèmes de calage vertical des données, notamment entre 1863 et 1920, période pendant laquelle le zéro hydrographique et le zéro du marégraphe n'était pas le même. Une étude est actuellement en cours pour vérifier la cohérence verticale de la série au cours de cette période.

Cette nouvelle série marégraphique historique permet également la redécouverte de tempêtes passées. La prise en compte de ces valeurs extrêmes, non quantifiées jusqu'alors, permet d'améliorer les estimations des niveaux extrêmes (BULTEAU et al., 2015). L'ensemble des évènements de tempêtes identifiés seront intégrés dans la base de données TEMPETES dont l'objectif est de capitaliser tout type d'information sur les événements de tempêtes et de submersions historiques (GILOY et al., 2018).

\section{Conclusions}

La valorisation des archives scientifiques en lien avec la marégraphie, longtemps restées oubliées, permet notamment la quantification de l'évolution séculaire du niveau moyen de la mer. Le projet de reconstruction de la série marégraphique de Saint-Nazaire a ainsi permis la digitalisation de plus de 80 années inédites de données horaires de hauteurs d'eau. A partir de cette nouvelle série, longue de plus de 150 ans, il a été estimé que les niveaux moyens à Saint-Nazaire augmentaient depuis 1863, à raison de $+1.03 \pm 0.05$ $\mathrm{mm} / \mathrm{an}$. Cette valeur tend à être plus faible que celles observées par ailleurs sur la façade atlantique sur des périodes comparables. Un travail en cours devrait permettre de vérifier la cohérence globale du référencement vertical de la série de Saint-Nazaire en s'appuyant notamment sur des comparaisons avec d'autres longues séries historiques (Brest, littoral Charentais, Newlyn, ...). L'impact de l'envasement régulier du puits de tranquillisation à Saint-Nazaire sur les niveaux moyens calculés sera investigué.

Le Shom dispose d'un volume important de mesures marégraphiques historiques, dont les plus anciennes datent du début du $19^{\text {ème }}$ siècle. Tout est mis en œuvre pour permettre la valorisation de ces archives scientifiques (inventaire, action de dématérialisation, digitalisation des données, ...).

Le travail de reconstruction systématique de ces séries marégraphiques permettra par ailleurs d'identifier des évènements de surcote non quantifiés jusqu'alors et ainsi d'apporter des éléments primordiaux pour l'étude séculaire des niveaux extrêmes. Ces informations sont essentielles pour une meilleure gestion des zones côtières.

\section{Remerciements}

Les auteurs tiennent ici à remercier le MTES/DGPR et la région Pays de la Loire pour le financement de la reconstruction de la série marégraphique de Saint-Nazaire. SONEL a contribué aux activités de data rescue (achat d'un scanner A0 à défilement, financement du scannage d'environ 8000 documents marégraphiques, étude préalable de vectorisation de données de hauteurs d'eau issues de registres et de marégrammes). 


\section{Thème 7 - Risques côtiers}

\section{Références bibliographiques}

BRADSHAW E., RICKARDS L., AARUP T. (2015). Sea level data archaeology and the Global Sea Level Observing System (GLOSS). GeoResJ, Vol. 6, pp 9-16. http://dx.doi.org/10.1016/j.grj.2015.02.005

BULTEAU T., IDIER D., LAMBERT J., GARCIN M (2015) How historical information can improve estimation and prediction of extreme coastal water levels: application to the Xynthia event at La Rochelle (France). Nat. Hazards Earth Syst. Sci., Vol. 15, pp 1135-1147. https://doi.org/10.5194/nhess-15-1135-2015

CAZENAVE A., LlOVEL W. (2010). Contemporary Sea Level Rise. Annu. Rev. Mar. Sci., Vol. 2, pp 145-173. https://doi.org/10.1146/annurev-marine-120308-081105

DOUGLAS B.C. (1991). Global sea level rise. J. Geophys. Res., Vol. 96, pp 69816992. https://doi.org/10.1029/91JC00064

FERRET Y. (2016). Reconstruction de la série marégraphique de Saint-Nazaire. Rapport n²7 Shom/DOPS/HOM/MAC, 122 p.

GILOY N., DULUC C-M., FRAU R., FERRET Y., BULTEAU T., MAZAS F., SAUZEAU T. (2018). La base de données TEMPETES : un support pour une expertise collégiale et interdisciplinaire des informations historiques de tempêtes et de submersions. XV ème JNGCGC, La Rochelle.

GOURIOU T. (2012). Evolution des composantes du niveau marin à partir

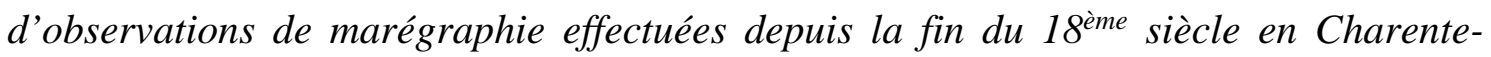
Maritime. Thèse Univ. de La Rochelle, 490 p. URL : https://tel.archives-ouvertes.fr/tel-00818425 GOURIOU T., MARTIN MIGUEZ B., WÖPPELMANN G. (2013). Reconstruction of a two-century long sea level record for the Pertuis d'Antioche (France). Continental Shelf Research, Vol. 61-62, pp 31-40. https://doi.org/10.1016/j.csr.2013.04.028

LATAPY A., HÉQUETTE A., POUVREAU N., WEBER N. (2017). Reconstruction of sea level changes in Northern France for the past 300 years and their relationship with the evolution of the coastal zone. COAST 2017. https://doi.org/10.13140/RG.2.2.14180.07041

PONS F. (2008). Améliorations récentes apportées au logiciel NUNIEAU pour la numérisation des marégrammes papiers. $\mathrm{X}^{\mathrm{èmes}}$ JNGCGC, Sophia Antipolis, pp 615-624. https://doi.org/10.5150/ingcgc.2008.059-P

POUVREAU N. (2008). Trois cents ans de mesures marégraphiques en France : outils, méthodes et tendances des composantes du niveau de la mer au port de Brest. Thèse

Université de La Rochelle, 468 p. URL : https://tel.archives-ouvertes.fr/tel-00353660

WÖPPELMANN G., MARCOS M., COULOMB A., MARTIN MIGUEZ B., BONNETAIN P., BOUCHER C., GRAVELlE M., SIMON B., TIPHANEAU P. (2014). Rescue of the historical sea level record of Marseille (France) from 1885 to 1988 and its extension back to 1849-1851. J. Geod., Vol. 88: pp 869-885. https://doi.org/10.1007/s00190-014-0728-6 